\title{
A Well-Define Titanium Complex for Free-Radical and Cationic Photopolymerizations Under Visible Light and Photoinduction of Ti-based Nanoparticles
}

Louise Breloy, Vlasta Brezová, Jean-Pierre Malval, Agustin Rios de Anda, Julie Bourgon, Takashi Kurogi, Daniel J. Mindiola, Davy-Louis Versace 


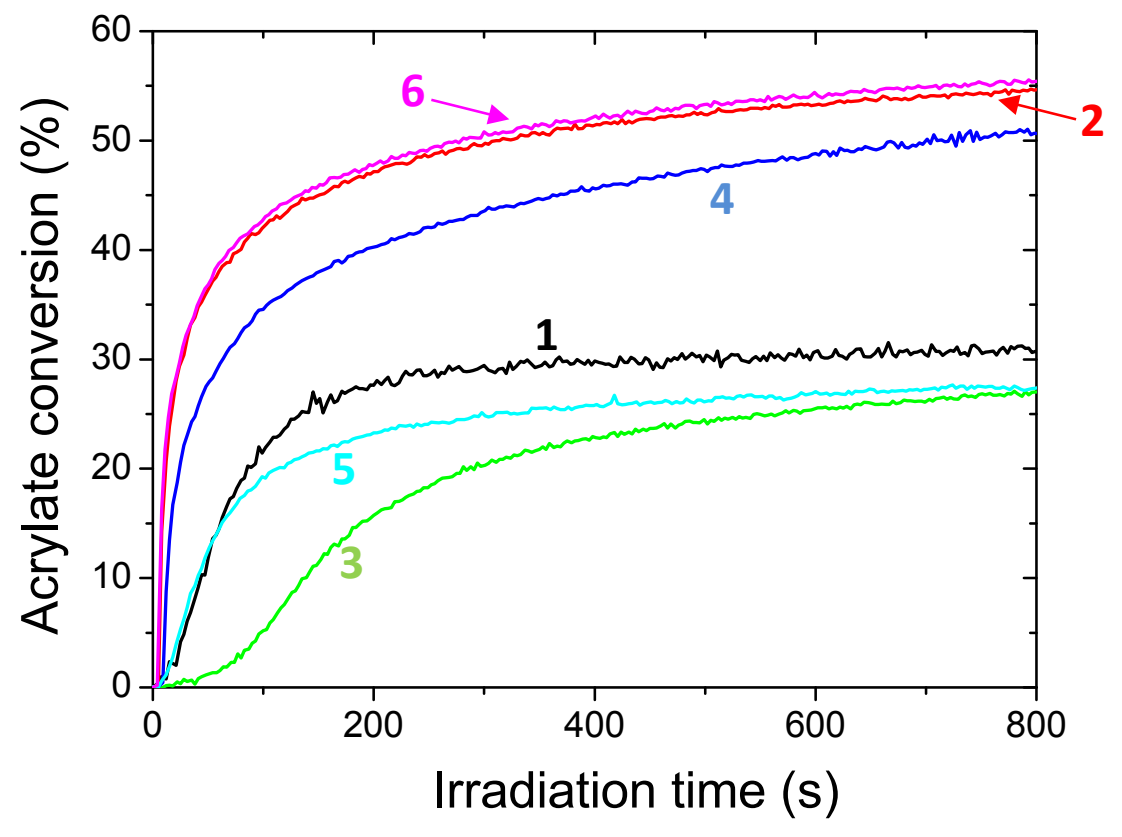

Figure S1. Free-radical photopolymerization of TMPTA in the presence of ITX/Trithiol (1\% / 3\%, w/w) upon 1) LED@385nm (12.5 mW/cm²) under air 2) LED@385nm $\left(12.5 \mathrm{~mW} / \mathrm{cm}^{2}\right)$ in laminate, 3) LED@405nm (12.5 mW/ $\left.\mathrm{cm}^{2}\right)$ under air, 4) LED@405 nm $\left(12.5 \mathrm{~mW} / \mathrm{cm}^{2}\right)$ in laminate, 5) LED@405nm (100 mW/ $\left.\mathrm{cm}^{2}\right)$ under air and 6) LED@405nm $\left(100 \mathrm{~mW} / \mathrm{cm}^{2}\right)$ in laminate. Thickness $=12 \mu \mathrm{m}$.

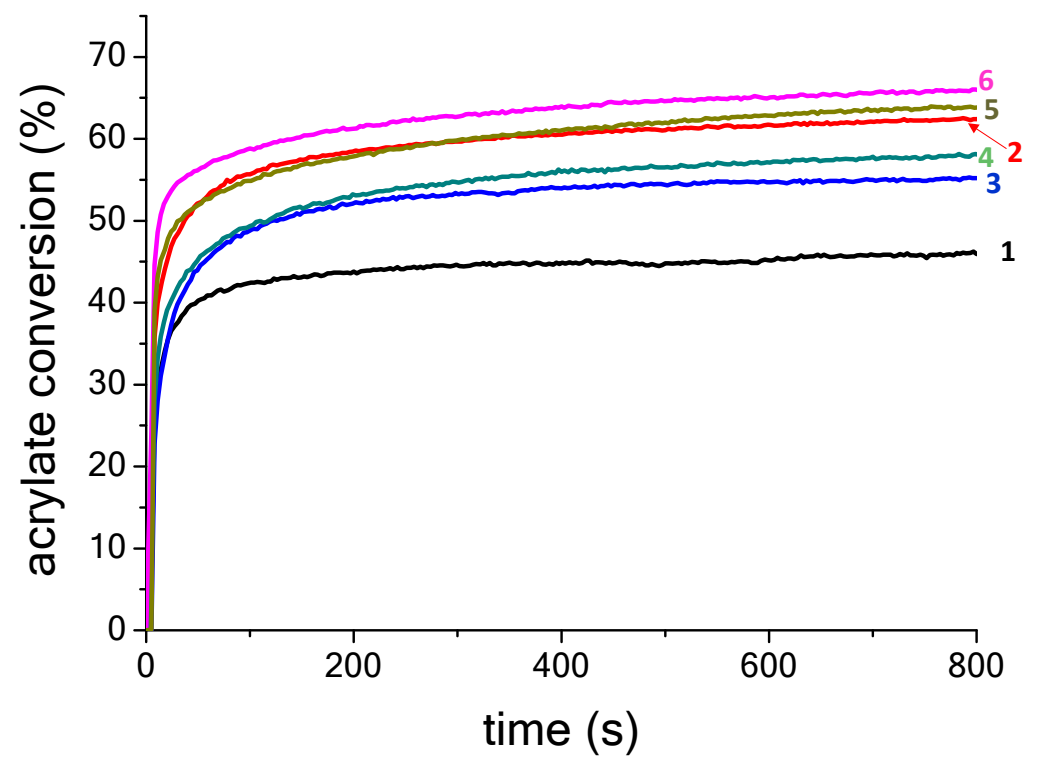

Figure S2. Free-radical photopolymerization of TMPTA in the presence of ITX/MDEA (1\% / 3.7\%,w/w) upon 1) LED@385nm (12.5 mW/cm²) under air 2)LED@385nm (12.5 mW/cm²) in laminate, 3) LED@405nm (12.5 mW/cm²) under air, 4) LED@405 nm (12.5 mW/ $\left.\mathrm{cm}^{2}\right)$ in 
laminate, 5) LED@405nm $\left(100 \mathrm{~mW} / \mathrm{cm}^{2}\right)$ under air and 6) LED@405nm $\left(100 \mathrm{~mW} / \mathrm{cm}^{2}\right)$ in laminate. Thickness $=12 \mu \mathrm{m}$.

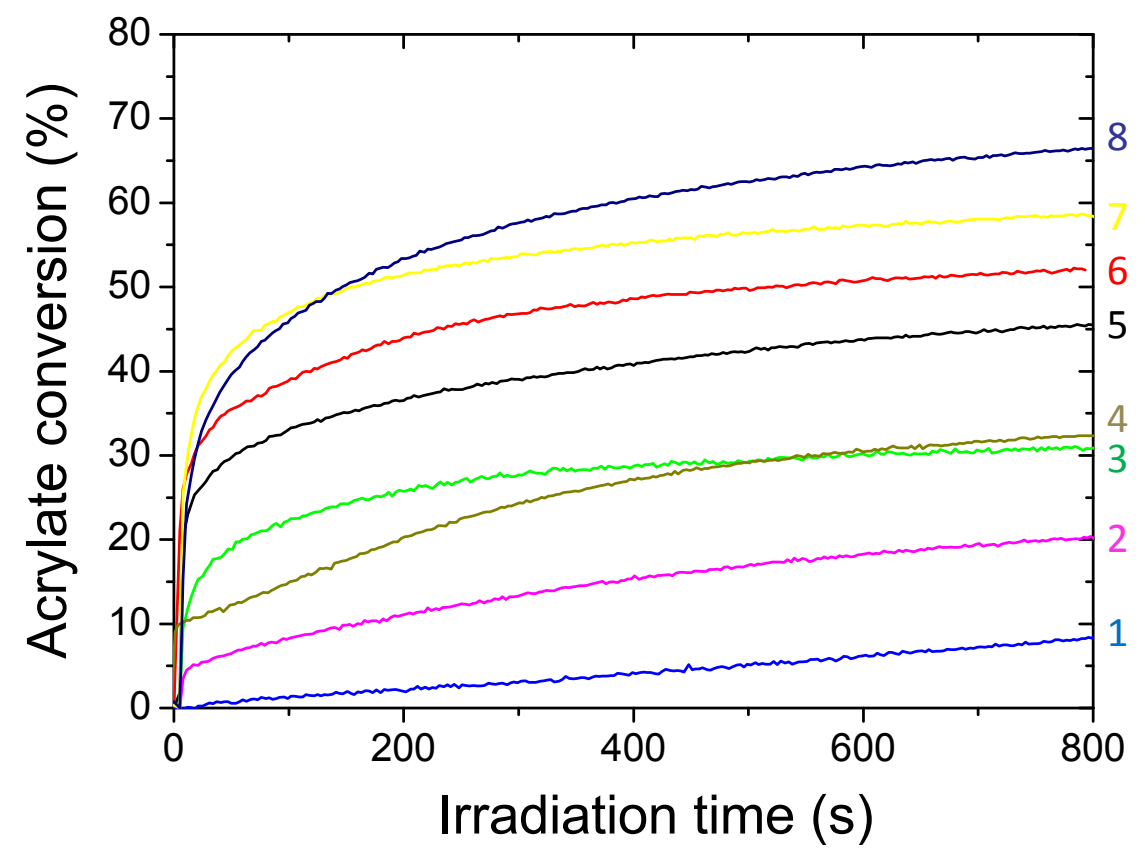

Figure S3. Free-radical photopolymerization of TMPTA in the presence of 1) Iod/Trithiol (3.7\% / 3\%, w/w) under air upon LED@385 nm $\left.\left(12.5 \mathrm{~mW} / \mathrm{cm}^{2}\right), 2\right)$ TiQ/Iod/Trithiol (1\% / 3.7\% / 3\%, w/w/w) under air upon LED@385 nm (12.5 mW/ $\left.\left.\mathrm{cm}^{2}\right), 3\right) \mathrm{TiQ} / \operatorname{Iod}(1 \% / 3.7 \%$, $\mathrm{w} / \mathrm{w})$ in laminate upon LED@385 nm $\left.\left(12.5 \mathrm{~mW} / \mathrm{cm}^{2}\right), 4\right)$ TiQ/Iod/Trithiol (1\% / 3.7\% / 3\%, w/w/w) under air upon LED@405 nm $\left(100 \mathrm{~mW} / \mathrm{cm}^{2}\right)$, 5) TiQ/Iod $(1 \% / 3.7 \%$, w/w) in laminate upon LED@405 nm $\left.\left(12.5 \mathrm{~mW} / \mathrm{cm}^{2}\right), 6\right) \mathrm{TiQ} / \mathrm{Iod}(1 \% / 3.7 \%$, w/w) in laminate upon LED@405 nm (100 mW/cm²), 7) TiQ/Iod/Trithiol (1\%/3.7\% / 3\%,w/w/w) in laminate upon LED@385 nm (12.5 mW/cm²) and 8) TiQ/Iod/Trithiol (1\%/3.7\% / 3\%,w/w/w) in laminate upon LED@405 nm $\left(100 \mathrm{~mW} / \mathrm{cm}^{2}\right)$. Thickness $=12 \mu \mathrm{m}$. 


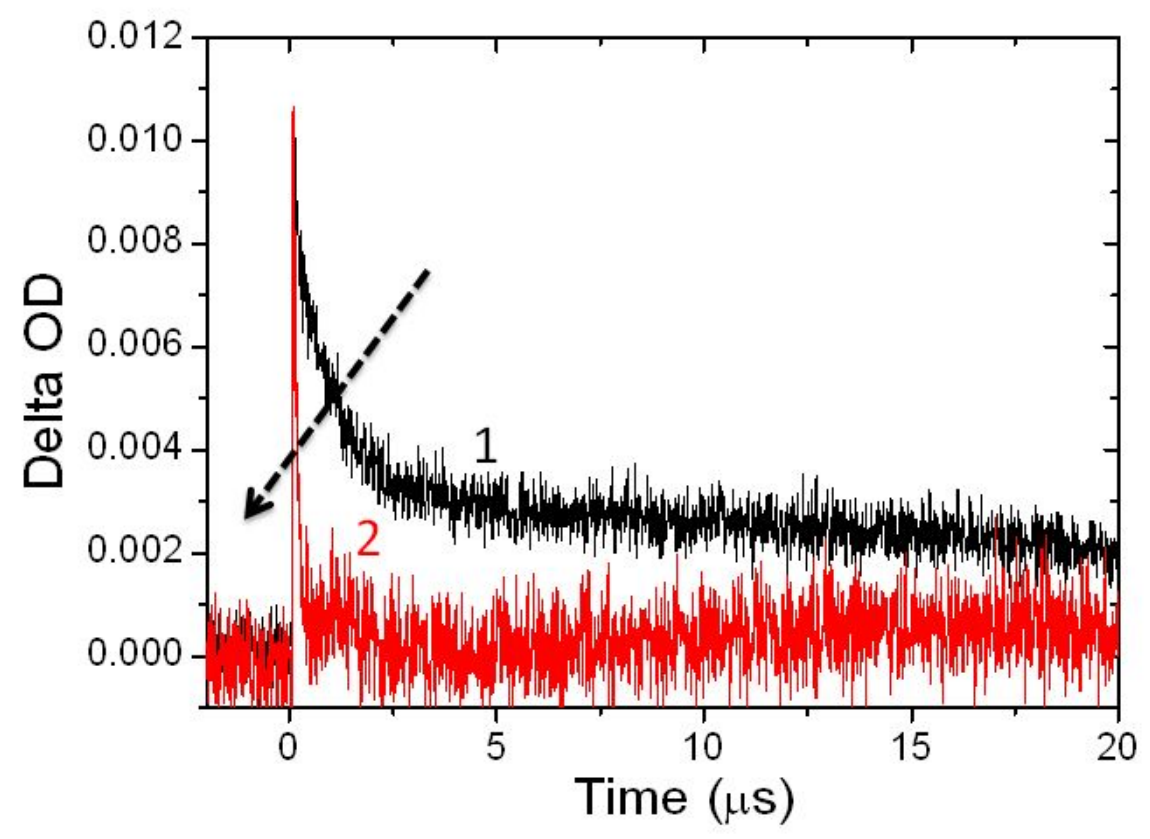

Figure S4. Decay traces of TiQ triplet state $\left({ }^{3} \mathrm{TiQ}\right)$ at $440 \mathrm{~nm}$ after a laser pulse $\left(\lambda_{\text {ex }}=385\right.$ $\mathrm{nm}$ ) of argon (1) and $\mathrm{O}_{2}(2)$ - satured toluene solution of TiQ.

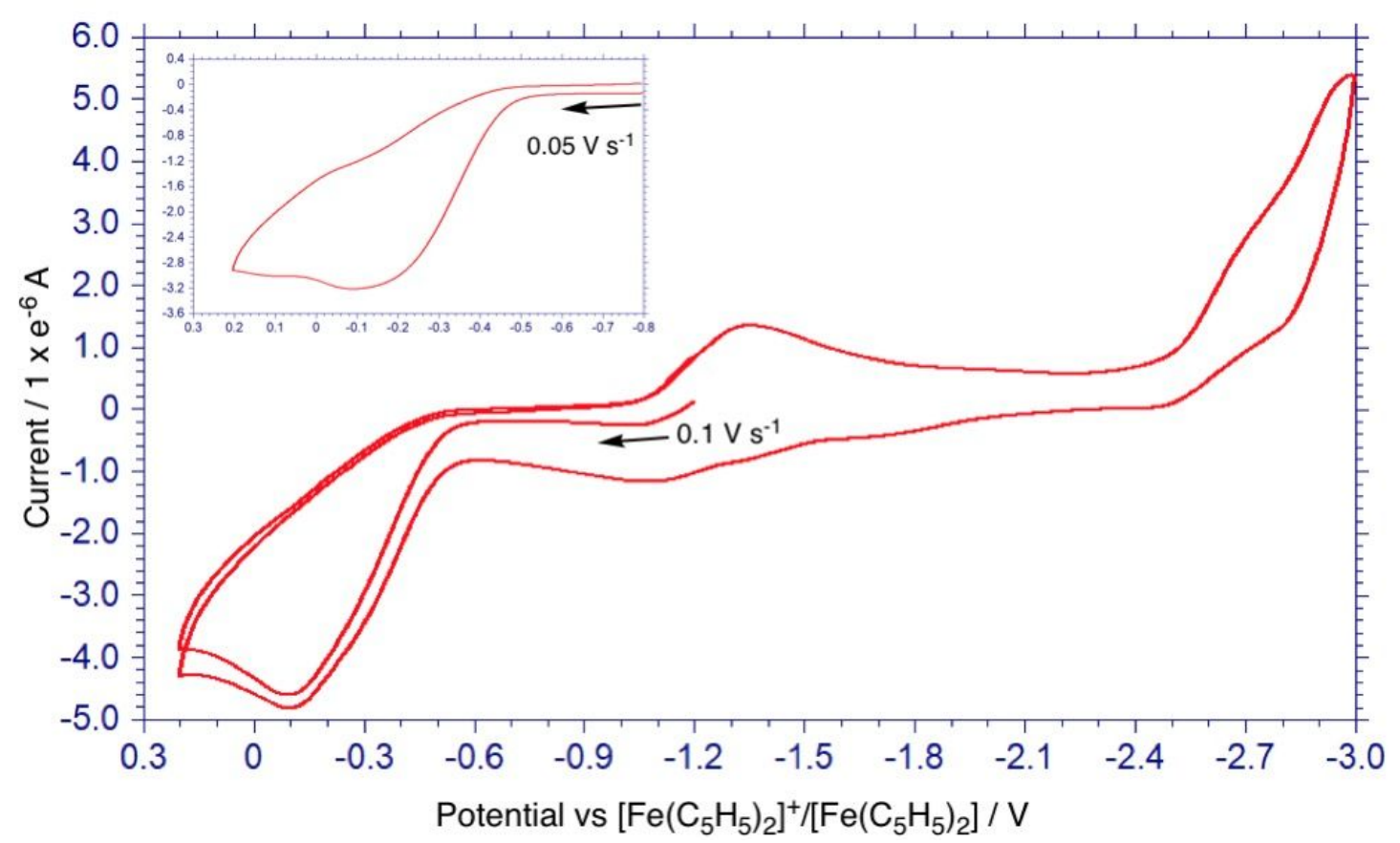

Figure S5. Cyclic voltammetry of TiQ in $\left[{ }^{n} \mathrm{Bu}_{4} \mathrm{~N}\right]\left[\mathrm{PF}_{6}\right] / \mathrm{THF}(0.2 \mathrm{M}$ electrolyte, room temperature, under $\mathrm{N}_{2}$ ). 

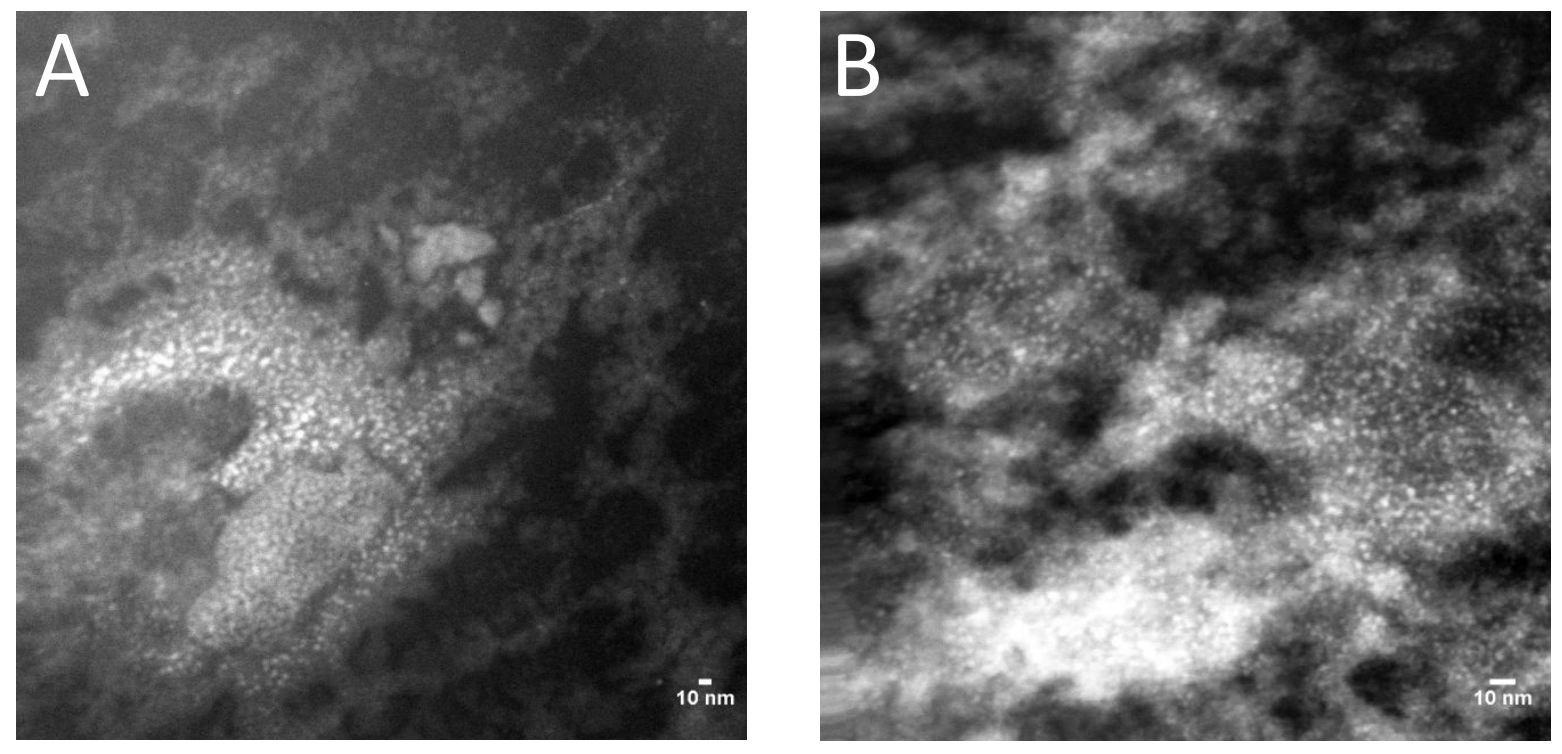

Figure S6. HAADF-STEM images of Ti NPs inside coatings from A) TiQ/Iod/EPOX system upon a LED@385 nm exposure (44 mW/ $\left.\mathrm{cm}^{2}\right)$ under air and B) EPOX/TMPTA blend $(50 \% / 50 \%, w / w)$ in the presence of TiQ/Iod/Trithiol upon a LED@405 nm exposure (100 $\mathrm{mW} / \mathrm{cm}^{2}$ ) under air. Irradiation time $=800 \mathrm{~s}$. 